\title{
PROGRAMA DE SAÚDE VOCAL PARA EDUCADORES: AÇÕES E RESULTADOS
}

\author{
Vocal health program for educators: actions and results
}

\author{
Maria Lúcia Oliveira Suzigan Dragone ${ }^{(1)}$
}

\begin{abstract}
RESUMO
Tema: a voz do professor tem sido foco de estudos nas últimas duas décadas devido à alta ocorrência de alterações vocais nesta classe profissional, assim, reforçou-se a necessidade dos professores participarem de ações para garantir saúde vocal. Poucos são os estudos na literatura que descrevem programas e seus resultados. Procedimentos: Descrever um Programa de Saúde Vocal desenvolvido no período 2002 a 2005, para educadores de ensino público (infantil e fundamental) do interior do Estado de São Paulo, composto por grupos básicos de voz oferecendo conhecimento teórico prático de cuidados vocais, com triagem da qualidade da voz dos participantes; grupos avançados buscando reorganização dos processos de fonação e do uso vocal em sala de aula. Resultados: em média $56 \%$ dos educadores inscritos frequentaram as ações; $62,9 \%$ das vozes apresentavam distúrbios na triagem vocal com maioria em grau discreto; no início dos grupos avançados $100 \%$ dos educadores participantes referiram mais de 3 sintomas associados ao uso vocal, e após, somente $45 \%$ deles ainda de 4 a 13 sintomas; os dados de autopercepção vocal revelaram baixos escores de impacto da voz nas atividades profissionais. Conclusão: a descrição revelou a necessidade de ajustes constantes do programa para alcançar seus objetivos. A baixa participação às ações pode estar relacionada à presença de impacto discreto da voz nas atividades profissionais, fato a ser mais bem investigado no futuro, e o benefício constatado objetivamente da participação dos educadores nos grupos avançados de voz foi a diminuição na quantidade de sintomas vocais.
\end{abstract}

DESCRITORES: Saúde do Trabalhador; Voz; Educação

\section{INTRODUÇÃO}

É inegável que na época atual, vista como a era da informação, a voz assume uma importância enorme na maior parte das funções profissionais e possui riscos ocupacionais diferenciados segundo cada contexto laboral e cultural. Um dos profissionais apontado consistentemente como de risco vocal é o professor, seguido por cantores, advogados e religiosos ${ }^{1,2}$.

Uma extensa revisão bibliográfica sobre voz profissional ${ }^{3}$ destaca as prioridades de Prevenção e Saúde Ocupacional (OSH - Occupational Safety and Health) relata que as pesquisas com a voz do professor enfocam principalmente a tentativa de entender melhor a relação da demanda de trabalho

(1) Fonoaudióloga; Docente do Ensino Superior do Centro Universitário de Araraquara, UNIARA, Araraquara, SP; Doutora em Educação pela Universidade Estadual Paulista UNESP, Araraquara,SP.

Conflito de interesses: inexistente e dos distúrbios vocais, sob o foco do uso diário, em jornadas semanais, falando em diferentes situações. Algumas conclusões já podem ser consideradas como válidas como aquelas relacionadas ao uso vocal em fala com alta intensidade e por longo tempo como fator desencadeante de um efeito negativo para a saúde vocal dos professores, e sobre a necessidade de treinar os professores para garantir boa saúde vocal. Sabe-se também que mesmo tendo conhecimento de como cuidar de suas vozes os professores podem não conseguir preservar suas vozes ao se defrontarem com os eventos inesperados da sala de aula, que algumas vezes tornam impossível o controle de seu comportamento vocal. Ao correlacionar-se estes dados, o autor sugere que cooperações entre sistemas de saúde ocupacional, de formação de professores e das instituições nas quais ocorre o trabalho docente seriam de crucial importância para o desenvolvimento de programas de saúde vocal para esta classe profissional. 
Aspectos epidemiológicos de saúde vocal dos professores foram estudados por um grupo de pesquisadores americanos ${ }^{4}$ cujos dados apontaram que os professores representavam $2 \%$ da população americana empregada e representavam também $16,4 \%$ dos pacientes que buscavam ajuda por problemas de voz no centro de Medicina de lowa e de Salt Lake City. Este mesmo grupo avançou a pesquisa com diversos profissionais investigando por intermédio de questionários seus aspectos vocais. Os resultados destes estudos mostraram que os professores referiram mais problemas de voz do que não professores (67\% contra $33 \%)$, marcadamente conduzindo a profissão a ser de risco para a presença de rouquidão. Outro grupo de pesquisadores americanos comparou professores e não professores ${ }^{5}$, e evidenciaram que os professores têm maior quantidade de sintomas vocais, percebem mudanças na qualidade vocal após uso da voz, e limitação profissionais por causa da voz do que o grupo de não professores. Na Irlanda, 244 professoras responderam a um questionário de autopercepção vocal ${ }^{6}$ contribuindo para a obtenção dos seguintes dados: $27 \%$ sentiam alteração vocal, $53 \%$ referiram problemas intermitentes de voz e somente $20 \%$ não apontaram esta alteração. A importância destes dados está na compreensão de que um problema intermitente pode se tornar crônico, e expõe a necessidade de intervenções preventivas nesta população. Os autores deste texto consideram a implantação de medidas que minimizem os fatores de risco vocal entre os professores, como: diminuição de carga horária e mais oportunidades de períodos de repouso vocal. Porcentagens e recomendações semelhantes foram encontradas também na Itália ${ }^{7}$ quando $48 \%$ dos professores pesquisados apresentaram alterações vocais frequentes na voz e $42 \%$ raras alterações, porém existentes.

Há mais de uma década as pesquisas brasileiras também foram gradualmente aprofundadas na tentativa de compreender melhor a problemática vocal do professor, suas causas e consequências ${ }^{8}$. Estudos sobre a ocorrência de alterações de voz entre professores brasileiros trazem números bastante expressivos, como os dados obtidos entre professores de ensino fundamental ${ }^{9}$, com $52 \%$ dos participantes referindo alterações vocais que foram comprovadas por análise perceptivo-auditiva feita por profissionais fonoaudiólogos. Presença de queixas vocais, incômodos presentes durantes a fala, e de autopercepção de professores sobre a presença de rouquidão foram referidas por $75 \%$ dos professores de ensino infantil e fundamental da rede Municipal de Ensino de São Paulo ${ }^{10}$, e em estudo da rede Municipal de Ensino de Belo
Horizonte ${ }^{11} 56 \%$ dos professores referiram piora da voz no decorrer do exercício profissional. A constatação de que a voz do professor sofre desgaste com o exercício profissional pode ser encontrada em um estudo longitudinal ${ }^{12}$ que avaliou vozes de professores de diferentes graus de ensino por duas vezes, num intervalo de dois anos, cujos resultados revelaram que $65,2 \%$ das vozes pioraram.

Percebeu-se que os professores têm necessidade de participar de programas de prevenção para cuidar de suas vozes, impedindo que rouquidões em decorrência do exercício profissional limitem sua participação no trabalho, os afastassem das aulas por meio de licenças muitas vezes dispendiosas aos mantenedores das escolas, ou, cheguem a impedimento do exercício profissional. Desta forma, passou-se a entender que se os professores conhecessem melhor a produção vocal, as possibilidades de seu uso em sala de aula, a viabilidade de modificá-la, de maximizá-la e principalmente as formas de cuidarem da voz traria aos professores menos problemas vocais no decorrer da carreira. Assim, muitos programas foram tomando forma, e sendo executados em benefício dos professores e de suas vozes.

Em alguns países como no Chile, a estruturação de programa de saúde vocal é favorecida pelo reconhecimento da disfonia como uma enfermidade profissional ${ }^{13}$. Os programas descritos pelos gestores de saúde ocupacional ampliaram as ações para espaços de discussão nas instituições de ensino, para realização de cursos que apresentem técnicas vocais para o bom uso da voz e intervenções primárias no meio ambiente, adequando-o a preservação da saúde vocal. Existindo essa amplitude de ação, ocorre uma visão integral do indivíduo e consequentemente um atendimento mais eficaz.

Exemplos de ações desenvolvidas em programas de saúde vocal e o relato dos benefícios obtidos constam na literatura ilustrando as diversas ações já desenvolvidas com grupos de professores. Um deles comparou um treinamento com utilização de amplificação sonora com abordagens envolvendo conhecimento de procedimentos de cuidados vocais e de realização de técnicas de ressonância e de respiração, realizado por pesquisadores americanos que revelaram benefícios significantes entre os professores treinados para utilizar amplificação sonora, e benefícios constatados nas medidas de função vocal e de impacto da voz na qualidade de vida nos grupos que utilizaram técnicas de ressonância e respiração ${ }^{14,15}$. Em outro estudo, pesquisadores irlandeses relataram a participação de alunos de pós-graduação em educação em um treinamento com dois tipos de abordagem: grupos com abordagem direta com melhores 
resultados nas medidas acústicas e sem mudanças no que diz respeito à autopercepção do impacto da voz, e grupo com abordagem indireta que não apresentou deterioração da voz ao final do treinamento (como o constatado no grupo controle), mas teve modificações na autopercepção de impacto vocal talvez associado ao conhecimento adquirido que aumentou a percepção das dificuldades vocais ${ }^{16}$. Semelhantes abordagens, uma com objetivo direto de mudar a função vocal por meio de técnicas para melhorar a produção vocal mais eficiente, e a outra oferecendo aos professores conhecimento sobre uso e cuidados vocais, mas incluindo o desenvolvimento de estratégias de minimizar riscos vocais obtiveram semelhantes resultados: os professores que receberam o treinamento vocal direto foram os que obtiveram melhores medidas de função vocal ao término dos programas ${ }^{17}$. Os resultados de um treinamento com exercícios de função vocal e conceitos de higiene vocal foram descrito por pesquisadores do Reino Unido apontando aumento do conhecimento sobre voz entre os participantes e diminuição na escala de severidade de sintomas vocal, mas, não houve melhora significante quanto aos índices de qualidade de vida e voz ${ }^{18}$. Uma descrição completa de um curso de aprimoramento vocal para professores universitários brasileiros, incluindo o uso de um manual sobre processos de emissão vocal e orientações sobre cuidados vocais, destaca a valorização pelos professores dos conteúdos e estratégias de preparo vocal, mas com preferência para aquelas de fácil execução, mais breves e com resultados imediatos na qualidade vocal ${ }^{19}$. Após 12 meses da realização de um curso para professores italianos do ensino infantil, abordando normas de ergonomia e exercícios de eficiência vocal segundo necessidades individuais, foi observado que eles ainda mantinham os exercícios, apresentavam boa ressonância e redução de tensão muscular, sem que fosse possível comprovar que não houve desgaste vocal ${ }^{20}$. A participação em um programa teórico-prático abordando uso vocal profissional entre professores brasileiros ${ }^{21}$ trouxe benefícios restritos de diminuição do uso da voz fora da atividade profissional e do uso de tons muito graves ou muito agudos durante as aulas, o que conduziu as pesquisadoras a sugerirem que intervenções outras mereceriam ampliação do foco das intervenções para esta classe profissional.

Estudos que abordaram a participação e a aderência dos professores nas ações propostas aparecem em menor ocorrência entre as publicações. Sobre esta questão, Roy e seus colaboradores ${ }^{14}$, referiram que os resultados do trabalho com professores que participavam de orientações sobre cuidados vocais poderiam ter sido melhores se a adesão fosse maior, pois, um terço dos participantes de um dos grupos não completou o programa de treinamento ${ }^{15}$. Dificuldades semeIhantes foram detectadas em um estudo brasileiro no qual houve dificuldade em conseguir a participação de professores em um curso de aprimoramento vocal ${ }^{19}$. Evidenciar a estrutura, os resultados e as dificuldades da realização de Programas Saúde Vocal na literatura oferece subsídios para que se compreenda melhor os acertos e as falhas destas intervenções.

Por esta razão, estruturou-se este texto que tem por objetivo descrever as ações realizadas e os resultados obtidos durante o Programa de Saúde Vocal de Educadores de uma rede municipal de ensino.

\section{APRESENTAÇÃO DO CASO}

Trata-se de um estudo descritivo analítico, estruturado no formato de relato de caso autorizado pela Secretaria Municipal de Educação de Araraquara-SP que em parceria com o Curso de Fonoaudiologia do Centro Universitário de Araraquara UNIARA promovem um Programa de Saúde Vocal do Educador.

A base de dados foi composta por: relatórios anuais do Programa de Saúde Vocal do Educador do período 2002 a 2005, cujo conteúdo aborda as diversas fases do projeto, as ações desenvolvidas e os resultados obtidos; e por dados provindos dos prontuários da Clínica UNIARA de Fonoaudiologia com a devida autorização da responsável pela instituição.

Todos os educadores que participaram das ações envolvendo algum tipo de avaliação individual sempre o fizeram após assinatura de Termo de Consentimento Livre e Esclarecido. O Teste de Igualdade de duas Proporções foi utilizado para comparar a proporção de respostas de duas determinadas variáveis e os seus níveis de significância, com p-valor igual a 0,05.

O projeto deste estudo foi aprovado pelo Comitê de Ética em Pesquisa do Centro Universitário de Araraquara ( $n^{\circ}$ 540/2006).

A Estruturação do Programa de Saúde Vocal A estruturação do programa foi realizada pela professora do Centro Universitário responsável pela disciplina de Voz e pelo estágio de Saúde Coletiva, houve sempre a colaboração das fonoaudiólogas e dos gestores da Secretaria de Educação do Município de Araraquara para atender as regras administrativas e as prioridades da instituição; e, a execução das ações foi realizada pelos alunos de últimos períodos do Curso de Fonoaudiologia. A solicitação desta parceria fundamentava-se nos 
dados de risco vocal entre professores usuários de voz no exercício profissional $1,2,4,6,10,11$; e a estruturação gradual do programa seguiu orientações difundidas na literatura ${ }^{3,14,15}$ sobre prevenção de saúde ocupacional em geral e vocal de professores.

Nesta Rede Municipal de Ensino todos os envolvidos nas ações educativas do ensino infantil e fundamental são denominados por educadores, e compuseram o foco das ações do Programa de Saúde Vocal sob solicitação da Secretaria de Educação de não priorizar somente os professores, pois todos educadores utilizavam a voz no contato educativo.

A saber, os educadores eram subdivididos em: gestores responsáveis pelas orientações do processo educativo realizadas com seus pares e pelo funcionamento da escola; professores atuantes no processo educativo formal de ensino infantil e fundamental; recreacionistas com atuação em atividades lúdicas em horários diferentes aos da educação formal; e berçaristas responsáveis pelo cuidado e estimulação de bebês até 2 anos.

As ações propostas foram divididas em dois tipos: grupos básicos e grupos avançados de voz. Os educadores foram convidados a participar das ações, realizavam inscrições confirmando a presença, recebiam um certificado de participação e tinham suas presenças controladas por serem ações consecutivas e a participação no grupo avançado dependia da participação prévia no grupo básico. Antes de iniciar os convites para os grupos avançados foram realizadas reuniões com os educadores indicados pela constatação de alteração vocal na triagem realizada durante o grupo básico, com explicações sobre as próximas atividades e solicitação de manifestação de interesse por escrito. As atividades ocorreram fora do horário de trabalho dos educadores segundo solicitações administrativas.

O conteúdo teórico dos Grupos Básicos envolvia o conhecimento sobre comportamentos vocais específicos ao trabalho docente, informações sobre produção e cuidados vocais; e, o conteúdo prático abordava treinamento de tarefas fonatórias básicas para aumento de resistência vocal e diminuição de tensão, conforme sugerido pela literatura ${ }^{13,14}$. Os grupos eram compostos por no máximo 50 educadores, que participavam de dois encontros de três horas, perfazendo uma carga horária total de seis horas. As vozes dos participantes foram registradas por gravador digital MD Sony MZ em duas amostras: emissão de vogal [a] prolongada e contagem de 1 a 20, configurando amostras para triagem vocal como critério de seleção para a formação dos Grupos Avançados de Voz. A análise desta amostra foi realizada por fonoaudióloga especialista em voz utilizando avaliação perceptivo-auditiva da qualidade vocal segundo Escala GRBASI estruturada inicialmente por Hirano em 1981, conforme descrito na literatura ${ }^{22}$, na qual se avalia o grau de rugosidade, soprosidade, astenia, tensão e irregularidade da voz, compondo o grau geral de alteração vocal $(G)$ em 4 graus de severidade: voz sem alteração quando o grau geral de rouquidão é igual a zero $\left(G_{0}\right)$; voz com alteração discreta quando seu grau geral é igual a um $\left(G_{1}\right)$; alteração moderada quando o grau geral é igual a dois $\left(G_{2}\right)$; e severa quando igual a três $\left(G_{3}\right)$. Em 2004 iniciouse o processo de estruturação dos Grupos Avançados de Voz, que envolveu um estudo detalhado de como viabilizar as ações. Como os educadores com vozes qualificadas como roucas moderadas $\left(G_{2}\right)$ ou severas $\left(G_{3}\right)$, durante a triagem, provavelmente encontravam-se em situação de dificuldade para utilizar suas vozes no exercício profissional, e provavelmente precisavam de uma atenção um pouco mais dirigida para as especificidades de seu problema vocal, foram privilegiados na convocação para participar dos primeiros Grupos Avançados de Voz. Posteriormente seriam chamadas também as educadoras com rouquidão discreta $\left(G_{1}\right)$.

Assim, estas educadoras foram convocadas a participar de reuniões com a fonoaudióloga do Centro Universitário, responsável pelo programa, com a finalidade de compreenderem os objetivos da nova ação e de declararem interesse em participar. Novamente, não houve possibilidade de liberação, das educadoras para esta atividade, em horários de atividade profissional. O local escolhido foi a Clínica Escola de Fonoaudiologia do Centro Universitário, pela viabilidade de horários, pós-trabalho das educadoras, e espaço físico condizente com as necessidades da ação.

A estruturação do Grupo Avançado previa um total de 10 horas de treinamento em grupo, subdivididas em cinco encontros de duas horas com no máximo cinco integrantes, e assim foi realizado o primeiro Grupo formado por educadoras com vozes classificadas em $\mathrm{G}_{3 .}$.

Todos participantes foram encaminhados para avaliação otorrinolaringológica, com médicos de seus convênios de saúde, e faziam avaliação completa no setor de Voz da Clínica Escola no início e no final das atividades. Nos encontros propiciava-se uma discussão aprofundada sobre o uso vocal em sala de aula na busca de estratégias que minimizassem o esforço durante a comunicação oral, e os exercícios escolhidos para treinamento abordavam tarefas fonatórias que favorecessem conforto vocal segundo o grau de distúrbio de voz do grupo, principalmente com exercícios de coordenação respiração $x$ facilitação de fonação $e$ ressonância. Orientações sobre busca de cuidados 
médicos e de indicação de terapia fonoaudiológica individual foram realizadas formalmente quando necessário. Formas semelhantes a esta proposição de abordagem da saúde vocal do professor podem ser encontradas nas publicações sobre programas de saúde vocal 16-18,20 que, embora com configurações diferenciadas, buscavam uma melhor produção vocal e a diminuição de riscos de piora vocal durante o exercício profissional.

No primeiro encontro dos grupos avançados foi respondido pelos educadores o Protocolo VAPP (Voice Activity and Participation Profile ${ }^{23}$ ) para que se conhecesse a autopercepção da severidade do problema de voz e a interferência da voz em atividades profissionais e sociais, segundo a avaliação dos próprios educadores. Utilizou-se uma tradução para o português disponibilizada no Grupo RAACCEV-SP ${ }^{24}$. Este protocolo é composto por 28 questões para auto-avaliação sobre qualidade da voz, impacto no trabalho, impacto na comunicação diária e profissional, impacto na emoção, impacto na limitação da atividade profissional e impacto na restrição trabalho. Os escores são obtidos por marcações em linha horizontal não graduada nas quais o limite à esquerda significa pior qualidade vocal (somente para a questão um sobre alteração de voz) ou nunca ocorre (em todas as demais questões envolvendo questionamento sobre a interferência da voz nas mais diversas situações), e o limite da direita é considerado o extremo oposto (pior qualidade vocal e sempre). $\mathrm{O}$ texto original do protocolo ${ }^{23}$ contém uma data base de escores obtidos por respostas de sujeitos com vozes normais e de sujeitos com vozes disfônicas, que embora sejam de cultura diferente oferece um apoio para uma avaliação qualitativa dos escores.

Os educadores, tanto no primeiro como no último encontro do Grupo Avançado, sinalizaram os sintomas vocais sentidos nos 15 dias anteriores, a partir de uma lista baseada em um estudo sobre sintomas presentes durante a fala que podem sinalizar problemas na fonação ${ }^{25}$, e que estabelece a presença de atrito vocal na presença três ou mais sintomas. A lista continha os seguintes sintomas: cansaço vocal, rouquidão, falhas na voz, perda da voz no final das frases, perda da voz no começo das frases, sensação de secura na garganta, incomodo na garganta, esforço para falar, sensação de aperto na garganta, necessidade de limpar a garganta (de pigarrear), sensação de corpo estranho na garganta, respiração curta enquanto fala, sensação de ardência ou queimação na garganta; dor na garganta, sensação de raspar na garganta.

\section{RESULTADOS}

\section{Sobre a participação nos Grupos Básicos}

No período de 2002 a 2005 foram realizados 14 grupos básicos com um total de 387 educadores distribuídos em grupos contendo em média 30 educadores, menores do que previsto por causa de ausências não justificadas de professores inscritos em alguns grupos, ou por inscrições abaixo do número de vagas. Os professores compuseram a maioria com $39,8 \%$ dos participantes (Tabela $1)$, seguidos por berçaristas $(30,7 \%)$, recreacionistas $(14,5 \%)$, agentes educacionais $(12,9 \%)$ e gestores $(2,1 \%)$. Embora o controle de inscritos e reais participantes não tenha sido realizado, segundo a percepção da equipe da Secretaria de Educação houve sempre defasagem entre números de inscritos e números de participantes, estimandose em aproximadamente $40 \%$ de ausências. Esta observação resultou em inúmeros ajustes quanto ao critério de divulgação das ações, forma de convites e de inscrição, na busca de melhorar o índice de participação dos educadores.

\section{Resultados da triagem vocal realizada no início dos Grupos Básicos}

As vozes de 372 educadores, $96 \%$ dos participantes dos grupos básicos, foram registradas com sucesso (vogal /a/ prolongada e contagem de $1 \mathrm{a}$ 20). A análise perceptivo-auditiva destas amostras foi realizada por fonoaudióloga especialista em voz utilizando escala GRBASI ${ }^{22}$. A Tabela 2 traz

Tabela 1 - Participantes dos grupos básicos

\begin{tabular}{lcc}
\hline EDUCADORES & $\mathbf{N}$ & $\%$ \\
\hline professores & 154 & 39,79 \\
berçaristas & 119 & 30,75 \\
recreacionistas & 56 & 14,47 \\
agentes educadionais & 50 & 12,92 \\
gestores & 8 & 2,07 \\
\hline total & 387 & 100 \\
\hline
\end{tabular}


Tabela 2 - Grau de alteração vocal dos educadores em triagem vocal

\begin{tabular}{|c|c|c|c|c|c|c|c|c|c|c|}
\hline \multirow{3}{*}{ Educadores } & \multicolumn{8}{|c|}{ Escala GRBASI } & \multirow{2}{*}{\multicolumn{2}{|c|}{ Total }} \\
\hline & \multicolumn{2}{|c|}{$\mathrm{G}_{0}$} & \multicolumn{2}{|c|}{$\mathrm{G}_{1}$} & \multicolumn{2}{|c|}{$\mathrm{G}_{2}$} & \multicolumn{2}{|c|}{$\mathbf{G}_{3}$} & & \\
\hline & $\mathbf{N}$ & $\%$ & $\mathbf{N}$ & $\%$ & $\mathbf{N}$ & $\%$ & $\mathbf{N}$ & $\%$ & $\mathbf{N}$ & $\%$ \\
\hline Gestores & 4 & 1,0 & 2 & 0,5 & 2 & 0,5 & 0 & 0,0 & 8 & 2,2 \\
\hline Professores & 42 & $11,0^{*}$ & 68 & 18,3 & 30 & 8,1 & 8 & 2,2 & 148 & 39,8 \\
\hline Recreacionistas & 42 & $11,0^{*}$ & 39 & 10,5 & 16 & 4,3 & 5 & 1,3 & 102 & 27,4 \\
\hline Berçaristas & 50 & $13,0^{*}$ & 46 & 12,4 & 16 & 4,3 & 2 & 0,5 & 114 & 30,6 \\
\hline Total & 138 & 37,0 & 155 & 41,7 & 64 & 17,2 & 15 & 4,0 & 372 & 100,0 \\
\hline
\end{tabular}

Teste Igualdade de duas proporções; $p$-valor $=0,05$

Tabela 3 - Dados sobre a participação nos grupos avançados de voz por educadores com vozes roucas severas (G3)

\begin{tabular}{lcccccccccc}
\hline \multirow{2}{*}{$\begin{array}{l}\text { Situação sobre } \\
\text { participação }\end{array}$} & \multicolumn{2}{c}{$\begin{array}{l}\text { Educadoras } \\
\text { convocadas }\end{array}$} & \multicolumn{3}{c}{ Presença na avaliação } & \multicolumn{3}{c}{ Frequência ao grupo } \\
\cline { 2 - 14 } & $\mathbf{N}$ & $\%$ & $\mathbf{N}$ & $\%$ & $\mathbf{N}$ & $\%$ & $\mathbf{N}$ & $\%$ & $\mathbf{N}$ & $\%$ \\
\hline Aposentada & 1 & 11,1 & - & - & - & - & - & - & - & - \\
Desistência da vaga & 1 & 11,1 & - & - & - & - & - & - & - & - \\
Incompatibilidade de horário & 1 & 11,1 & - & - & - & - & - & - & - & - \\
Aceite da convocação & 6 & $66,7^{\star}$ & 4 & 66,6 & 2 & 33,4 & 3 & 75 & 1 & 25 \\
\hline Total $(\mathrm{N}=9)$ & 9 & 100 & 4 & 44,4 & 2 & 22,2 & 3 & 33,3 & 1 & 11,1 \\
\hline
\end{tabular}

Teste Igualdade de duas proporções; $p$-valor $=0,05$

os dados relacionados ao grau geral de alteração (G): $62,9 \%$ dos educadores apresentaram vozes consideradas como alteradas, a maioria delas com rouquidão discreta, e somente $4 \%$ com rouquidão considerada severa. Na comparação entre os tipos de funções dos educadores somente entre professores, recreacionistas e berçaristas houve semeIhança significativa de ocorrência de vozes sem alteração.

\section{Sobre a participação dos educadores no Grupo Avançado}

O primeiro grupo de educadores foram aqueles com vozes rouco-severas. Importante ressaltar que até o momento da convocação havia somente nove educadoras com grau de alteração severa. Destas nove educadoras somente quatro (representando $44,4 \%$ das convidadas) iniciaram a participação no grupo avançado realizando a avaliação completa de voz, três delas tiveram participação parcial nas ações, e uma educadora somente teve aderência plena com $100 \%$ de freqüência (Tabela 3 ).

Novamente a baixa adesão e a real participação dos educadores foram analisadas tanto pela responsável pelo programa como pelos gestores da Secretaria de Educação e as seguintes possíveis causas foram delineadas: dificuldade pela distância entre o local de trabalho das educadoras e a Clínica Escola, frequência semanal em cinco encontros de duas horas, motivos particulares de saúde, tempo decorrido entre a triagem e a convocação (exemplo: caso da professora que já havia se aposentado); e falta de motivação dos educadores para participar da ação. Em comum acordo decidiu-se pela convocação de uma reunião com as educadoras dentro do horário de trabalho delas, com objetivo de explicar novamente a importância dos grupos avançados, motivando-as para o valor da voz nas relações com os alunos, e manteve-se a necessidade de que ao final da reunião as educadoras confirmassem o interesse na participação dos grupos. Decidiu-se também que a duração de cada encontro do Grupo Avançado seria reduzido para 90 minutos, mantendo-se os 5 encontros.

Em 2005 foram convocadas para esta reunião 54 educadoras cujas vozes foram classificadas como rouco-moderadas $\left(G_{2}\right)$ nas triagens realizadas até aquela data. Conforme exposto na Tabela 4, houve declaração de interesse por 28 educadoras (51\% das convocadas para a reunião inicial) formandose quatro grupos avançados de voz, sendo que 22 das educadoras inscritas iniciaram os grupos avançados realizando a avaliação completa de voz, mas, somente 17 (31\% das inicialmente convocadas) 
Tabela 4 - Participação das educadoras com grau de alteração vocal moderada (G2) nos grupos avançados de voz - 2005

\begin{tabular}{lcc}
\hline \multirow{2}{*}{ Especificação do envolvimento/participação } & \multicolumn{2}{c}{ Educadoras } \\
\cline { 2 - 3 } & $\mathbf{N}$ & $\%$ \\
\hline Convocadas para reunião inicial $-\mathrm{G}_{2}$ & 54 & 100 \\
Presentes na reunião inicial & 33 & 61 \\
Pontuaram interesse nos Grupos avançados & 28 & 51 \\
Compareceram na Avaliação Global da Voz & 22 & 40 \\
Participaram dos Grupos (+75\% de presença) & 17 & 31 \\
\hline
\end{tabular}

Teste Igualdade de duas proporções; $p$-valor $=0,05$

Tabela 5 - Laudos otorrinolaringológicos dos educadores dos grupos avançados

\begin{tabular}{lcc}
\hline \multirow{2}{*}{ Exame Otorrinolaringológico } & \multicolumn{2}{c}{ Educadores } \\
\cline { 2 - 3 } & $\mathbf{N}$ & $\%$ \\
\hline Sem exame & 3 & 17,7 \\
Sem alterações & 5 & 29,4 \\
Nódulos & 2 & 11,7 \\
Nódulos ou cisto & 2 & 11,7 \\
Fenda fusiforme & 1 & $5,9^{\#}$ \\
Fenda posterior & 1 & $5,9^{\#}$ \\
Fenda paralela & 1 & $5,9^{\#}$ \\
Hiperemia & 1 & $5,9^{\#}$ \\
Somente imagem sem laudo & 1 & $5,9^{\#}$ \\
\hline Total & 17 & 100 \\
\hline
\end{tabular}

Teste Igualdade de duas proporções; $p$-valor $=0,05$

participaram efetivamente o grupo avançado com frequência superior a $75 \%$ dos encontros. Estes dados sugerem que apesar da baixa participação entre os educadores com voz $\mathrm{G}_{2}$, o grupo avançado ficou configurado em um formato viável para a participação de educadores interessados. A convocação dos educadores com grau discreto de rouquidão ficou prevista para ser realizada em 2006, fora do período descrito neste estudo.

\section{Sobre os dados otorrinolaringológicos}

Todas as educadoras que compareceram à avaliação completa de voz no início dos grupos avançados foram encaminhadas para exame otorrinolaringológico. Entre as efetivas participantes dos grupos avançados somente 13 apresentaram laudo otorrinolaringológico, uma apresentou somente a imagem gravada em vídeo, e as demais não conseguiram agendar consulta médica antes do final do grupo. Os dados obtidos consolidaram a importância da participação destas educadoras no programa de saúde vocal e demonstraram que entre as vozes classificadas na triagem roucas moderadas havia, na sua maioria envolvimento de alterações funcionais, carentes de modificações do padrão fonatório para garantir saúde vocal.

Uma análise detalhada dos laudos otorrinolaringológicos (Tabela 5) revela que $29,4 \%$ das educadoras que participaram dos grupos avançados não tinham alterações laríngeas. Observou-se, em 47\% dos laudos otorrinolaringológicos a ocorrência de nódulos, cistos ou fendas.

\section{Sobre a avaliação do impacto da voz nas atividades profissionais durante os Grupos Avançados}

Os educadores do programa, ora descrito, responderam ao protocolo VAPP ao iniciarem os grupos avançados. As respostas das 17 educadoras participantes ofereceram dados intrigantes quando comparados com os valores médios encontrados pelos autores do protocolo entre disfônicos e não disfônicos (Tabela 6). A média dos escores obtidos pelos educadores revela interferência 
Tabela 6 - Escores VAAP obtidos durante os grupos avançados comparados com padrões entre vozes normais e entre vozes disfônicas ${ }^{23}$

\begin{tabular}{|c|c|c|c|c|}
\hline $\begin{array}{l}\text { Escore } \\
\text { máximo } \\
\text { possível }\end{array}$ & Categoria & $\begin{array}{c}\text { Escores } \\
\text { para vozes } \\
\text { normais }\end{array}$ & $\begin{array}{c}\text { Escores médios } \\
\text { entre educadores } \\
\mathrm{N}=17\end{array}$ & $\begin{array}{l}\text { Escores entre } \\
\text { disfônicos }\end{array}$ \\
\hline 10 & \multirow{2}{*}{$\begin{array}{l}\text { Percepção de alteração vocal } \\
\text { Interferência comunicação no } \\
\text { trabalho }\end{array}$} & 0,98 & 2,69 & 6,23 \\
\hline 40 & & 1,72 & 9,0 & 17,22 \\
\hline 120 & Interferência comunicação diária & 6,97 & 31,26 & 50,88 \\
\hline 40 & \multirow{2}{*}{$\begin{array}{c}\text { Interferência comunicação social } \\
\text { Interferência manifestação das } \\
\text { emoções }\end{array}$} & 1,25 & 6,2 & 12,47 \\
\hline 70 & & 2,57 & 16,36 & 27,33 \\
\hline 240 & \multirow{2}{*}{$\begin{array}{c}\text { Escore total } \\
\text { Limitação no trabalho por causa da } \\
\text { voz }\end{array}$} & 13,49 & 65,51 & 114,13 \\
\hline 30 & & 2,12 & 8,5 & 13,82 \\
\hline 30 & $\begin{array}{c}\text { Restrição no trabalho por causa da } \\
\text { voz }\end{array}$ & 1,19 & 4,47 & 13,03 \\
\hline
\end{tabular}

Tabela 7 - Número de sintomas vocais pré e pós-grupo avançado $(n=14)$

\begin{tabular}{cccccc}
\hline Número de & \multicolumn{3}{c}{ Pré } & \multicolumn{3}{c}{ Pós } & \multirow{2}{*}{ p-valor } \\
\cline { 2 - 5 } Sintomas & $\mathbf{N}$ & $\%$ & $\mathbf{N}$ & $\%$ & \\
\hline 0 & 0 & 0,0 & 2 & 14,3 & 0,142 \\
De 1 a 3 & 0 & 0,0 & 5 & 35,7 & $0,018^{*}$ \\
De 4 a 6 & 3 & 21,4 & 4 & 28,6 & 0,684 \\
De 7a 9 & 7 & 50,0 & 2 & 14,3 & $0,066^{\#}$ \\
De 10 a 13 & 4 & 28,6 & 1 & 7,1 & 0,157 \\
\hline
\end{tabular}

Teste Igualdade de duas proporções; $p$-valor $=0,05$

discreta da voz nas suas atividades: classificam suas vozes como discretamente alteradas $(2,69$ de escore máximo em 10,0), sentem a voz interferindo de forma discreta em todas situações de comunicação analisadas com escores atingindo de 15,5\% a $26 \%$ dos escores máximos possíveis, da mesma forma se comportaram os dados relacionados à limitação ou restrição de participação nas suas ações profissionais com escores entre $14,9 \%$ a $28 \%$ dos máximos possíveis.

\section{Sobre a avaliação da presença de sintomas vocais pré e pós-grupo avançados}

Fez-se a opção de analisar somente a quantificação de sintomas apontados pelos educadores, ao início e no final dos grupos avançados, com a finalidade de observar se ocorreriam modificações do quadro de atrito vocal (Tabela 7).

Foi possível obter respostas sobre sintomas vocais de 14 educadores para a análise pré e pósparticipação dos grupos avançados. Os dados
(Tabela 7) revelam que houve sensível diminuição da presença de sintomas vocais entre os participantes. Antes da participação nos grupos avançados não houve referência de três sintomas ou menos, somente a partir de 4 e até 13 sintomas apareceram nas respostas dos educadores. Ao final do Grupo avançado 55,5\% dos participantes referiram de 0 a 3 sintomas. Ou seja, antes todos os educadores estavam com sinais da síndrome de atrito vocal (mais de três sintomas) e no após os grupos, somente $45 \%$ deles ainda apresentavam mais de três sintomas vocais, porém, com diminuição significativa representativa da quantidade de sintomas.

\section{DISCUSSÃO}

Referências sobre dificuldades em conseguir total adesão de professores a programas de saúde vocal ou a processos de formação em voz e comunicação são relatadas na literatura internacional ${ }^{14,15}$ 
e nacional ${ }^{19}$, tal qual observado no desenvolvimento do programa ora descrito, sem que se tenha conseguido chegar a uma definição precisa sobre o que os conduz a isso, e, carecem de estudos mais aprofundados na compreensão dos fatores causais desta situação.

A ocorrência de $62,9 \%$ de alteração vocal entre os educadores constatada por análise perceptivo auditiva (Tabela 2), é semelhantes aos dados encontrados por Dragone et al ${ }^{12} \mathrm{e}$ aos dados de Roy et al ${ }^{5}$ obtidos pela referência de alterações vocais em estudo abordando a autopercepção dos professores; porém, encontram-se superiores aos dados de outros estudos com o mesmo foco ${ }^{7,9}$.

Segundo laudos otorrinolaringológicos (Tabela 5), a ausência de alterações laríngeas em parte das educadoras participantes dos grupos avançados, cujas vozes alteradas estariam então relacionadas à disfonia funcional justificou totalmente um treinamento para adequar as formas de emitir a voz com a finalidade de impedir a evolução do caso. Da mesma forma, a presença de nódulos, cistos e fendas associados a provável disfonia organofuncional sinalizam uso incorreto da fonação ${ }^{22}$, configuraram-se como casos passíveis de serem minimizados ou revertidos com fonoterapia ${ }^{22} \mathrm{e}$ com a participação em programas de saúde vocal envolvendo cuidados vocais e conhecimento sobre uso vocal em sala de aula tal qual foi realizado nos grupos avançados. Na verdade, reforçaram também correlações entre avaliação preceptivoauditiva e exames laringológicos, nas quais graus de alteração moderados podem sugerir presença de alterações laríngicas.

Os dados do Protocolo VAPP revelaram que os educadores permaneceram numa posição intermediária entre os dados obtidos com disfônicos e não disfônicos pelos autores do protocolo ${ }^{23}$. Tal fato pode sugerir a baixa percepção de alteração vocal e da interferência da voz nas atividades profissionais entre estes educadores como um fator contribuinte para a baixa adesão ao programa. Desta forma, ao não sentirem limitações nem restrições nas atividades profissionais por causa da voz e sequer percebem vozes alteradas, não sentem necessidade de melhorar seus padrões de voz para o trabalho, consequentemente, sem motivação para participar das atividades do Programa de Saúde Vocal. A auto-percepção sobre limitações de participação e restrições no trabalho por causa da voz merecem ser melhor investigada em futuras abordagens envolvendo esta classe profissional.
A diminuição significativa da quantidade de sintomas vocais consolidou que a participação dos educadores no programa, especificamente nos grupos avançados, gerou benefício importante para a preservação das vozes dos educadores: menos sintomas, menor atrito e maior saúde vocal; assim como foram percebidos outros benefícios em estudos descritos na literatura, como: diminuição de uso intenso da voz fora da atividade profissional e utilização de tons mais neutros durante as aulas ${ }^{21}$, melhoria de ressonância e de tensão muscular com diminuição de desgaste vocal ${ }^{20}$, melhora da função vocal ${ }^{17}$, entre outros.

\section{CONCLUSÃO}

O Programa de Saúde Vocal direcionado aos educadores da rede municipal de ensino de Araraquara-SP, no período de 2002 a 2005, foi uma ação de prevenção e de aprimoramento vocal comprovadamente necessária pelos dados que evidenciaram algum grau de distúrbio vocal na maioria das vozes triadas. Mesmo sendo a maioria das vozes com alterações discretas eram merecedoras de atenção para não piorarem frente à demanda vocal exigida pela profissão. As ações em grupos terapêuticos trouxeram benefícios com a diminuição da quantidade sintomas associados ao uso vocal, o que significa diminuição de atrito vocal e piora futura. A avaliação da voz nas atividades de vida, segundo a autopercepção dos educadores participantes dos grupos avançados revelou índices baixos de impacto, sem limitação e restrição significantes às atividades profissionais. Em especial, este dado merece atenção, pois favorece a compreensão dos problemas de adesão e de participação dos educadores nas ações propostas pelo programa de saúde vocal: eles não sentem limitações, logo, não se interessam em aprimorar estes aspectos. Fatores múltiplos podem mascarar a percepção dos educadores sobre a relevância da voz no trabalho docente, e merecem ser mais bem investigados.

A descrição realizada ressalta a necessidade constante de reavaliação das ações contidas em um programa de saúde vocal, nos quais, os resultados objetivados somente se consolidarão com a participação ativa dos educadores, que por sua vez ocorre a partir da percepção de que o investimento de tempo e atenção irá gerar benefícios imediatos no contexto profissional. 


\begin{abstract}
Background: in the last two decades teacher's voice has been the target of many researches due to the high incidence of voice disturbances in this professional class; therefore, the need for these professionals to take part in actions that ensure the vocal health has been reinforced. Few papers in the literature describe vocal health programs and their results. Procedures: describe a Vocal Health Program of Educators from public schools (kinder garden and elementary schools) from the countryside of São Paulo inland, developed from 2002 to 2005, that was made up by basic voice group to offer essential theoretical and practical knowledge about vocal care, including the participants' vocal screening; advanced voice group to reorganize the phonation process and the voice use in classroom. Results: the average of $56 \%$ of total enrolment participated in the actions; $62.9 \%$ of voice disturbance is the result of vocal screening, most of it with discreet vocal deviation; $100 \%$ of participants referred 3 or more voice symptoms at the beginning of advanced voice group, and after the group had finished, $45 \%$ still had 4 to 13 symptoms; data about vocal self-perception show low scores in vocal impact in professional activities. Conclusion: the description showed the need for constant regulation of the program in order to reach its goals. The low participation in actions might be related to the discreet impact of voice in professional activities, although this fact requires further investigation. The highest measured benefit of the participation in the advanced vocal groups was the reduction in the volume $e$ of vocal symptoms.
\end{abstract}

KEYWORDS: Occupational Health; Voice; Education

\section{REFERÊNCIAS}

1. Koufman J, Isaacson G. The spectrum of vocal dysfunction. Otolaryngol Clin N Am. 1991; 24:985-8. 2. Verdolini K, Ramig L. Review:occupational risks for voice problems. Log Phon Vocol. 2001; 26(1):37-46.

3. Vilkman E. Ocupattional safety and health aspects of voice and speech professions. Folia Phoniatr. Logop. 2004; 56:220-53.

4. Smith E, Gray SD, Dove H, Kirchner L, Heras H. Frequency and effects of teachers' voice problems. J Voice. 1997; 11(1):81-7.

5. Roy N, Merril RM, Thibeault S, Gray SD, Smith EM. Voice disorders in teachers and general population: effects on work performance, attendance and future career choices. J Speech Lang Hear Res. 2004; 47:542-52.

6. Munier C, Kinsella R. The prevalence and impact of voice problems in primary school teachers. Occup Med. 2008; 58:74-6.

7. Bellia S, Serafino L, Luca N, Farruggia E, Bellia $M$. Incidenza della disfonia nel personale docente della scuola. G Ital Méd LAvErg. 2007; 29(3):613-4. 8. Dragone MLS, Behlau M. A fonoaudiologia brasileira e a voz do professor: olhares científicos no decorrer do tempo. Fonoaudiol Bras. 2006; 4:6-8. 9. Oliveira ML, Abraão CV, Silva RML, Aragão NRTL, Camargo Z. Triagem vocal em professores da Rede de Ensino: $1^{\text {a }}$ a $4^{\text {a }}$ séries na cidade de Três Rios-RJ. Rev. CEFAC. 2005; 7(4):509-13.
10. Vaz ACN, Trentini AL, Campos ASC, Melissopoulos CBG, Zoppelo DL, Carvalho LR et al. Ocorrência de queixas vocais em professores da Rede Municipal de Educação da cidade de São Paulo. IV Congresso Internacional de Fonoaudiologia e III Encontro Ibero - Americano de Fonoaudiologia. Palácio das Convenções do Anhembi, São Paulo. Anais. São Paulo: Sociedade Brasileira de Fonoaudiologia; 1999. p. 396.

11. Jardim R, Barreto SM, Assunção AV. Condições de trabalho, qualidade de vida e disfonia entre docentes. Cad. Saúde Pública. 2007; 23(10):2439-61.

12. Dragone MLS, Sicchiroli SC, Reis RA, Behlau M. Desgaste vocal do professor: um estudo longitudinal. Rev Soc Bras Fonoaudiol. 1999; 3(5):50-6.

13. Oyarce LAS. Comparación de parámetros acústicos de la voz en una muestra de profesoras disfônicas y um grupo sano. Cienc. Trab. 2005; 7(15):31-6.

14. Roy N, Weinrich B, Gray SD, Tanner K, Toledo $\mathrm{SW}$, Dove $\mathrm{H}$, et al. Voice Amplification versus vocal hygiene instruction for teachers with voice disorders: a treatment outcomes study. J Speech Lang Hear Res. 2002; 45:625-38.

15. Roy N, Weinrich B, Gray SD, Tanner K, Stemple JC, Sapienza CM. Three Treatments for teachers with Voice Disorders: a randomized clinical trial. J Speech Lang Hear Res. 2003; 46:670-88. 
16. Duffy OM, Hazlett DE. The impact of preventive voice care programs for training teachers: a longitudinal study. J Voice. 2004; 18(1):63-70.

17. Pasa G, Oates J, Dacakis G. The relative effectiveness of vocal hygiene training and vocal function exercises in preventing voice disorders in primary school teachers. Logoped Phoniatr Vocol. 2007; 32:128-40.

18. Gillivan-Murphy $P$, Drinnan MJ, O'Dwyer TP, Ridha $\mathrm{H}$, Carding P. The effectiveness of a voice treatment approach for teachers with self-reported voice problems. J Voice. 2006; 20(3):423-31.

19. Bovo R, Galceran M, Petruccelli J, Hatzopoulos S. Vocal problems among teachers: evaluation of a preventive voice program. J Voice. 2007; 21(6):705-22.

20. Grillo MHMM. The impact of a vocal improvement course in a speech language and hearing science prevention context. Pró-Fono. 2004; 16(2):159-68.
21. Simões-Zenari M, Latorre MRDO. Mudanças em comportamentos relacionados com o uso da voz após intervenção fonoaudiológica junto a educadoras de creche. Pró-Fono. 2008; 20(1):61-6. 22. Behlau M; Madazio G, Feijó D, Pontes P. Avaliação de voz. In: Behlau M, organizador. Voz: o livro do especialista. Rio de Janeiro: Revinter; 2001. p. 85-245.

23. Ma EPM, Yiu EML. Voice activity and participation profile: assessing the impact of voice disorders on daily activities. J Speech Lang Hear Rev. 2001; 44:511-24.

24. Behlau M, Lobo F, Caifa M. Tradução do Protocolo VAPP. [texto didático para RACC Reciclagem e Atualização Clínico-Científica. Centro de Estudos da Voz]. São Paulo: CEV; 2004.

25. Sapir S, Keidar A, Mathers-Schimidt B. Vocal attrition in teachers: survey findings. Eur $\mathrm{J}$ Disord Commun. 1993; 28(2):177-85.
http://dx.doi.org/10.1590/S1516-18462010005000059

RECEBIDO EM: 15/11/2009

ACEITO EM: 05/01/2010

Endereço para correspondência:

Maria Lúcia Oliveira Suzigan Dragone

Av. Prof. Augusto César, 902

Araraquara - SP

CEP: $14801-240$

E-mail: mldragone@uol.com.br 